\title{
Uniaxial stress studies of the Be pair bound exciton absorption spectrum in silicon
}

\author{
M O Henry ${ }^{\dagger}$, Keith A Moloney $\div$ J Treacy $\div \S$, F J Mulligan $\dagger$ and E C \\ Lightowlerst \\ $\div$ School of Physical Sciences, National Institute for Higher Education. Glasnevin. Dublin \\ 9. Ireland \\ $\div$ Wheatstone Physics Laboratory. Kings College. Strand. London WC2R 2LS. UK
}

Received 27 July 1984 , in final form 10 September 1984

\begin{abstract}
We report the results of preliminary uniaxial stress studies of the beryllium pair isoelectronic bound exciton absorption spectrum in silicon. Our results confirm that the exciton behaves as an isoelectronic acceptor.
\end{abstract}

\section{Introduction}

Recent spectroscopic studies of silicon in the near infrared have been devoted largely to isoelectronic bound exciton systems. Watkins et al (1984) list the majority of the systems reported to date. The variety of isoelectronic centres discovered has produced a rich vein of material for both experiments and theoretical study. In general, results obtained broadly confirm the basic theoretical framework developed by Hopfield et al (1966) for isoelectronic bound excitons in III-V and II-VI compound semiconductors. A comprehensive review of this subject is given by Dean and Herbert (1979). The basis of the Hopfield et al (1966) model is that the exciton binding centre sequentially captures the electron and hole. If the electron (hole) is trapped first and a hole (electron) subsequently bound in the coulombic field of the first particle then the exciton formed is classified as an isoelectronic acceptor (donor). In general, a bound exciton system can be classified as either acceptor-like or donor-like on the basis of the nature of the vibronic sidebands produced in the optical spectra (Hopfield et al 1966). Direct experimental evidence for the acceptor or donor nature of a bound exciton in the form of a series of effectivemass-like excited states is rare, being confined to one example in $\mathrm{GaP}$ (Cohen and Sturge 1977 ) and one example in Si (Wagner and Sauer 1982). This scarcity of data is partly due to the fact that many isoelectronic centres exist at low concentrations, so that absorption spectroscopy often fails to reveal their presence. It is noteworthy that in the two examples cited above luminescence excitation spectroscopy using tunable lasers was required to reveal the excited state structures.

In this study we return to one of the first isoelectronic bound exciton systems identified in $\mathrm{Si}$, the beryllium pair centre (Henry et al 1981). The Be pairs form a split interstitial about a silicon site in the crystal, and on the basis of magnetic field splittings have been assigned $(100\rangle$ axial symmetry (Henry et al 1981). Uniaxial stress data in $\S$ Visiting from the Regional Technical College, Tralee, Co Kerry, Ireland. 
luminescence at $4.2 \mathrm{~K}$ (to be published) appear to favour $\langle 111\rangle$ axes for the Be pairs. This discrepancy will be fully discussed in a future publication; for the present study it is not critical, as we explain below. The Be pair centres can be produced at concentrations in the region of $10^{16} \mathrm{~cm}^{-3}$ and a series of excited states are clearly evident in absorption and in luminescence excitation (Thewalt et al 1982). Although the isoelectronic bound exciton was tentatively identified as donor-like, the absorption spectrum could not be fitted to a hydrogenic series of donor-like states. A recent study by Labrie et al (1984) reveals that transitions in the far infrared from the ground state of the bound exciton into its excited states indicate acceptor-like behaviour by this system. We present in this study the results of preliminary uniaxial stress measurements on the near-infrared exciton absorption lines which support the designation of the bound exciton at Be-pair centres in Si as acceptor-like.

\section{Experimental}

The samples used in this study were prepared in the manner described by Henry et al (1981). For the absorption measurements a $200 \mathrm{~W}$ tungsten halogen lamp was used as a radiation source. A Spex 1704 spectrometer and North Coast Ge detector were used to respectively analyse and detect the radiation transmitted by the samples. A microcomputer was used in conjunction with the spectrometer controller to control the operation of the spectrometer and to record and process the data. All measurements reported here took place at $85 \mathrm{~K}$ in a stream of cold nitrogen vapour using a cryostat of the Maeda (1965) type. The stress apparatus was based on the use of lead weights and a lever arm, and the system was calibrated using a load cell. The absorption features of interest fall in the energy range $1070-1120 \mathrm{meV}$ which encompasses a group of strong water-vapour absorption lines. To eliminate these features from our spectra a background spectrum was recorded with the sample removed from the optical path and the sample spectra normalised with respect to this background.

\section{Results}

An absorption spectrum recorded at $85 \mathrm{~K}$ is shown in figure 1 . The general features are essentially the same as those observed at $2 \mathrm{~K}$ (Thewalt et al 1982). The line widths at $85 \mathrm{~K}$ are a factor of about four greater than at $2 \mathrm{~K}$, and the weakest lines detectable at $2 \mathrm{~K}$ do not show up in the $85 \mathrm{~K}$ spectrum. Nevertheless, the four main lines in the absorption spectrum, labelled 1 to 4 in order of increasing energy in figure 1, are sufficiently sharp and well resolved to enable high-stress effects to be examined. Line 1 in our notation corresponds to the electric-dipole-allowed $\mathrm{A}$ line transition in the conventional notation used for isoelectronic bound excitons (Dean and Herbert 1979, and references therein). The forbidden $B$ line transitions are not observed in absorption, but show up clearly in luminescence at low temperatures (Henry et al 1981). Note that the width of line 1 (the A line) at $85 \mathrm{~K}, 2 \mathrm{meV}$, is comparable with the total spread of energies in the exciton A and B line manifold (Henry et al 1981). This broadening of the spectral lines precludes any study of the fine structure which may exist in the spectra due to the electron-hole exchange and/or axial strain splittings of the exciton states. We are obliged, therefore, to exclude consideration of the axial symmetry of the Be pairs in this study and to limit our analysis to large-stress effects. In the limit of large stresses, the 


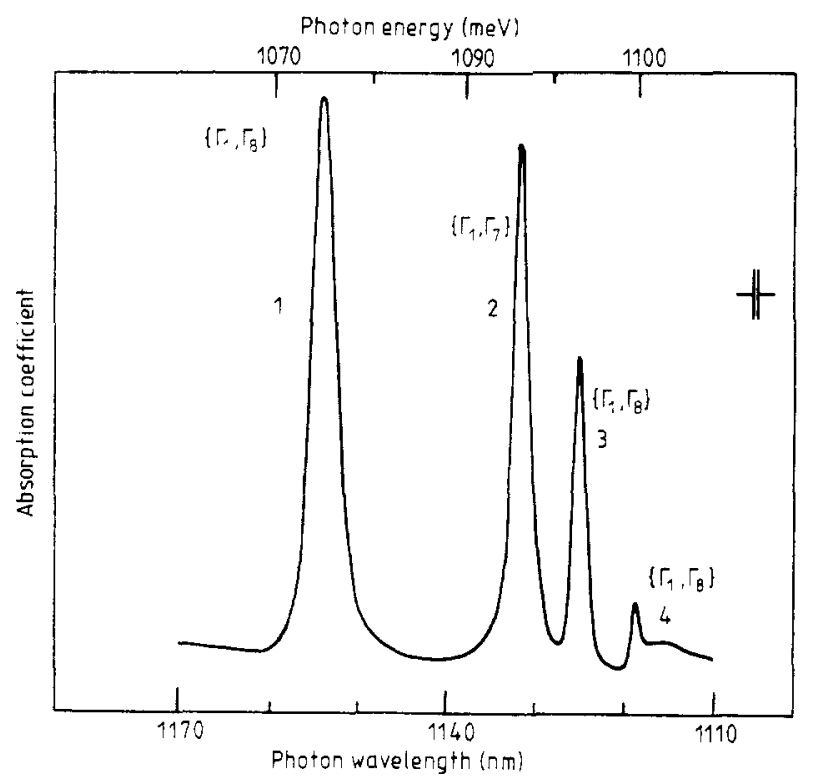

Figure 1. The absorption of $\mathrm{Si}: \mathrm{Be}$ at $85 \mathrm{~K}$. The peak of the strongest line in the spectrum corresponds to an absorption coefficient of $0.15 \mathrm{~cm}^{-1}$. The four lines in the spectrum are labelled according to the electron-hole pairs which constitute the exciton, after the model of Labrie et al (1984).

magnitude of the axial strain splittings becomes negligible and, for the data analysis, the exciton binding centre can be assumed to possess the $\bar{T}_{d}$ point group of a silicon site.

Figure 2(a) shows a series of spectra recorded for uniaxial stresses along a $\langle 111\rangle$ crystal axis. Line 1 splits into three components, whose relative intensities are stress dependent. Similar behaviour is observed for line 1 for stresses along the $\langle 001\rangle$ and $\langle 011\rangle$ crystal axes, as shown in figure $2(b)$ and $2(c)$ respectively. Line 2 does not split for any stress while lines 3 and 4 both show two components under all stress directions. There is evidence of considerable interaction among several subcomponents for all three stress directions. Figure 3 shows the energy positions of the stress-split components of all lines as a function of the applied stress for the three principal stress directions.

\section{Discussion}

Before analysing the details of the stress data we provide a summary of the results implicit in the study of Labrie et al (1984). These authors propose acceptor-like nature for the Be pair bound exciton in silicon. Thus the electron is tightly bound and assumed to occupy a $\Gamma_{1}$ state, as is observed for the ground state of substitutional donor electrons (Kohn and Luttinger 1955). The hole of the exciton is considered to be weakly bound in a series of effective-mass-like states by the coulombic field of the electron. We assume that for the ground state of the bound exciton (line 1 in our absorption spectrum) the hole occupies the usual $\Gamma_{8}$ ground state. In the manner originally adopted by Kirczenow (1977) for bound exciton systems, we identify line 1 with the creation of an electronhole pair denoted by $\left\{\Gamma_{1}, \Gamma_{8}\right\}$. Similarly, the remaining lines in the absorption spectrum 


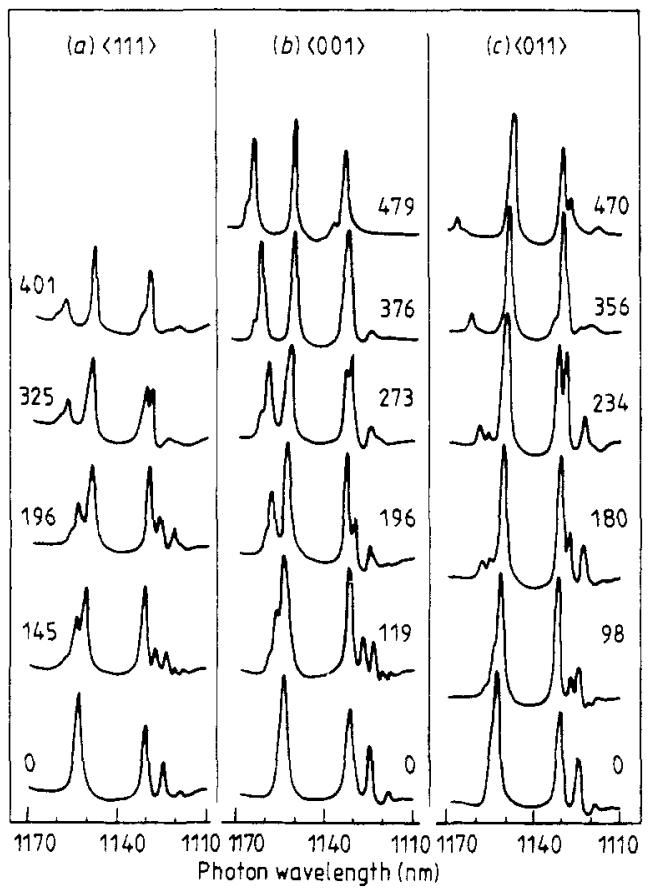

Figure 2. Absorption spectra of $\mathrm{Si}$ : $\mathrm{Be}$ at $85 \mathrm{~K}$ for uniaxial stresses (marked on curves in $\mathrm{MPa})$ along $(a)\langle 111\rangle,(b)\langle 001\rangle$ and $(c)\langle 011\rangle$ crystal axes.

in figure 1 are labelled $\left.\left\{\Gamma_{1}, \Gamma_{7}\right\}, \Gamma_{1}, \Gamma_{8}\right\}$ and $\left\{\Gamma_{1}, \Gamma_{8}\right\}$ in order of increasing energy. This labelling scheme treats the electron and hole as quasi-independent particles. This is certainly an incomplete description for the lowest exciton manifold. However, as we pointed out above, the effects produced by the exchange interaction between the

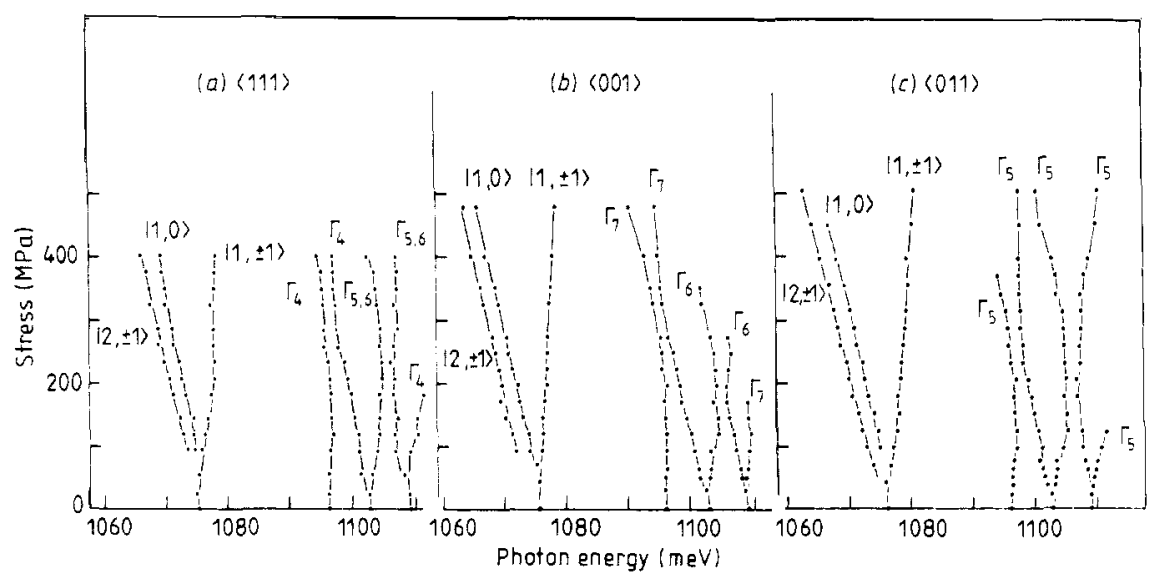

Figure 3. Stress splittings of the $\mathrm{Si}$ : Be absorption lines at $85 \mathrm{~K}$ for stresses along $(a)\langle 111\rangle$, (b) $\langle 001\rangle$ and $(c)\langle 011\rangle$ crystal axes. Line 2 and the components of lines 3 and 4 are labelled with the irreducible representation of the hole involved in the exciton states observed. The components of line 1 are labelled in the $\left|J, M_{j}\right\rangle$ notation for the exciton. 
electron and hole and the axial strain at the Be pair centre are barely detectable for the exciton ground state manifold in the present study. Furthermore, there is no evidence in the study of Labrie et al (1984) for exchange or strain splittings in the exciton excited state manifolds. For our present purposes, therefore, it is adequate to describe the exciton states in terms of quasi-independent electron-hole pairs. A unique feature of the foregoing discussion is the assignment of the first excited state manifold of the exciton to an electron-hole pair involving a $\Gamma_{7}$ hole. There is considerable theoretical support for the existence of a low-lying $\Gamma$, state for effective mass acceptors in silicon (Lipari and Baldereschi 1978). Raman scattering studies in boron-doped silicon by Wright and Mooradian (1967) and Cherlow et al (1973) and infrared absorption studies by Chandrasekhar et al (1973) provide experimental evidence for the existence of this state. In this study further evidence for a low-lying $\Gamma_{7}$ hole state is presented and the acceptorlike nature of the Be pair bound exciton is confirmed.

The most striking feature of the data presented in figures 2 and 3 is the behaviour of line 2 which does not show any evidence of stress-induced broadening or splitting. This specific result and the general trends in the stress splittings for lines 2.3 and 4 can be explained on the basis of effective-mass-like acceptor states. Since the electron in each case occupies a non-degenerate $\Gamma_{1}$ state, stress-induced splittings are not allowed for the electron. Thus, whichever splittings are observed are due to the behaviour under stress of the hole states. The influence of uniaxial stresses on the effective-mass acceptors is well documented theoretically (Rodriguez et al 1972) and experimentally (Chandrasekhar et al 1973). We present in table 1 the results which apply to $\Gamma_{6}, \Gamma_{7}$ and $\Gamma_{8}$ holes (the only classifications allowed for acceptors in $\left.T_{d}\right) . \Gamma_{8}$ hole states are seen to produce two components for all stress directions, whereas the $\Gamma_{6}$ and $\Gamma_{7}$ holes (both Kramers doublets) cannot be split by any stress. Accordingly, we can positively rule out the involvement of a $\Gamma_{8}$ hole in the exciton state corresponding to line 2 in our absorption spectrum. There is not sufficient information in this study to determine whether line 2 involves a $\Gamma_{7}$ or $\Gamma_{6}$ hole. However, as Labrie et al (1984) have pointed out, the separation of lines 1 and 2 strongly favours a $\Gamma_{7}$ assignment to the hole. Based on this assumption, the information given in table 1 can be used to label line 2 for all stresses, as shown in figure 3 . Table 1

Table 1. Effects of uniaxial stress on acceptor states in silicon.

\begin{tabular}{llll}
\hline & $\langle 111\rangle$ & $\langle 001\rangle$ & $\langle 011\rangle$ \\
$\bar{I}_{d}$ & $C_{3,}$ & $D_{2 d}$ & $C_{3,}$ \\
\hline$\Gamma_{6}$ & $\Gamma_{\downarrow}$ & $\Gamma_{6}$ & $\Gamma_{5}$ \\
$\Gamma_{*}$ & $\Gamma_{\downarrow}$ & $\Gamma_{-}$ & $\Gamma_{5}$ \\
$\Gamma_{*}$ & $\Gamma_{4}, \Gamma_{5}+\Gamma_{6}$ & $\Gamma_{6}, \Gamma_{7}$ & $\Gamma_{5}, \Gamma_{5}$ \\
\hline
\end{tabular}

can also be used to explain the occurrence of interactions between various components of the absorption lines under stress. For example, under $\langle 011\rangle$ stress the $\Gamma_{7}$ hole of line 2 transforms according to $\Gamma_{5}$, while both components of line 3 also transform as $\Gamma_{5}$. The expected interaction between pairs of $\Gamma_{5}$ levels as they approach is clearly evident in figures $2(c)$ and $3(c)$. We have used the information in table 1 to label all components of lines 2,3 and 4 for $\langle 011\rangle$ stresses in figure $3(c)$.

Line 2 interacts with the lower-energy component of line 3 for all stresses, and this enables the line 3 components to be assigned on the basis of the data in table 1 . Thus, for $\langle 111\rangle$ stress, the lower-energy component of line 3 is labelled $\Gamma_{4}$ and the upper component 
$\left(\Gamma_{5}+\Gamma_{6}\right)$. Similarly, for $\langle 001\rangle$ stress the lower and upper components of line 3 are designated $\Gamma_{7}$ and $\Gamma_{6}$, respectively. In order to designate the components of line 4 , evidence of interactions, if any, between components of lines 3 and 4 is required. There is a gradual drop in the intensity of the line 4 components as stress is increased, which is probably caused by the hole state involved becoming less tightly bound in the exciton. Nevertheless, the limited data available suggest that under both $\langle 111\rangle$ and $\langle 001\rangle$ stresses the components of lines 3 and 4 which approach interact weakly. We conclude, therefore, that the ordering of the components of line 4 is reversed compared with line 3 , and figure 3 is labelled accordingly. A similar reversal of the ordering of some hole state sublevels occurs for acceptors in silicon (Chandrasekhar et al 1973).

We now consider in outline the case of line 1 . The stress data reveal three components, with stress-dependent relative intensities. In the absence of polarisation data, the analysis of the relative intensities is postponed. The effects of uniaxial stress on isoelectronic bound excitons was originally analysed by Morgan and Morgan (1970) and Onton and Morgan (1970). Under a uniaxial compression the $J=1$ and $J=2$ states of the exciton form five levels denoted in a $\left|J . M_{j}\right\rangle$ notation by $\left.|1, \pm 1\rangle,|1,0\rangle, 2, \pm 2\right\rangle,|2, \pm 1\rangle$ and $|2,0\rangle$ in order of decreasing energy. Transitions into the $\langle 2, \pm 2\rangle$ and $|2,0\rangle$ states are forbidden, so three transitions are expected in absorption. Of these, transitions into the $|2, \pm 1\rangle$ state are allowed only by virtue of mixing with the $|1, \pm 1\rangle$ state. Onton and Morgan (1970) show that the energy separation between the states $|1, \pm 1\rangle$ and $|1,0\rangle$ equals the basic $\Gamma_{8}$ hole state splitting of the $J=1$ exciton state under stress. The separation between the $|1,0\rangle$ and $|2, \pm 1\rangle$ states at large stresses is approximately $75 \%$ of the exchange splitting between the $J=1$ and $J=2$ levels. Our data for $\mathrm{Si}$ : Be correlate closely to the theory outlined above. In figure 3 we have labelled the three components of line 1 with the appropriate $\left|J, M_{i}\right\rangle$ labels. Thus, the behaviour of line 1 is qualitatively explained. We must point out that stress measurements in luminescence at $4.2 \mathrm{~K}$ provide a wealth of detail not evident in the present study. This detail will be presented in a quantitative analysis of the exciton $A$ and $B$ line system which is at present underway.

Finally, we extract estimates of the deformation potentials $b$ and $d$ for the $\Gamma_{8}$ holes involved in lines 1, 3 and 4 of the Be pair bound exciton absorption spectrum. These coefficients are defined by the relations (Laude et al 1971)

$$
\Delta_{\{001\rangle}=2 b\left(s_{11}-s_{12}\right) T \quad \Delta_{\langle 111\rangle}=\left(d / V_{3}\right)\left(s_{44}\right) T
$$

where $\Delta_{\{001\rangle}$ and $\Delta_{\{111\}}$ denote the energy separation of the $\Gamma_{8}$ hole sublevels under $\langle 001\rangle$ and $\langle 111\rangle$ stresses, respectively, for an applied stress $T . s_{11}, s_{12}$ and $s_{44}$ are the silicon elastic compliance constants. For lines 3 and 4 , the separation of the stress-split components gives a direct measure of the hole state splitting. For line 1, the separation between the two highest-energy components describes the effective $\Gamma_{8}$ hole state splitting for the $J=1$ exciton state under large stresses (Onton and Morgan 1970). Using published values of the elastic compliance constants at liquid nitrogen temperatures (Laude et al 1971), our data yield the values of $b$ and $d$ shown in table 2 . In the table our results are compared with the corresponding free-exciton parameters obtained by Laude et al (1971). The values obtained for $d$ are very close to the free-exciton values, while the values of $b$ show a reduction of at most $50 \%$. This result for $\mathrm{Si}: \mathrm{Be}$ is in contrast with an order-of-magnitude reduction in the value of $b$ (their $D_{\mathrm{u}}$ ) obtained by Onton and Morgan (1970) for the GaP : Bi bound exciton. The latter is an example of an isoelectronic donor for which the deeply bound hole is clearly non-effective-mass-like. Although our data provide approximate estimates only, they clearly favour the isoelectronic acceptor model for the Be pair bound exciton in silicon. 
Table 2. Deformation potentials $b$ and $d$ for $\Gamma_{8}$ holes in the $\mathrm{Si}$ : Be bound exciton.

\begin{tabular}{lllll}
\hline & Line 1 & Line 3 & Line 4 & Free exciton $^{(\mathrm{a})}$ \\
\hline$b(\mathrm{eV})$ & 1.2 & 1.1 & 1.1 & $2.10 \pm 0.10$ \\
$d(\mathrm{eV})$ & 3.8 & 4.8 & 4.9 & $4.85 \pm 0.15$ \\
\hline
\end{tabular}

The values estimated for $b$ and $d$ are accurate to $\sim 10 \%$.

(a) Laude et al. (1971).

\section{Conclusion}

The results we have presented in this study provide a qualitative analysis of the behaviour under stress of the $\mathrm{Si}: \mathrm{Be}$ bound exciton absorption spectrum. The bound exciton excited states are shown to be consistent with isoelectronic acceptor-like behaviour for the $\mathrm{Be}$ pair bound exciton. In particular, the data support the assignment of a $\Gamma_{7}$ hole state to the first excited state manifold of the bound exciton.

\section{Acknowledgments}

Keith Moloney acknowledges the support of the Research and Postgraduate Studies Committee of NIHE Dublin, and John Treacy acknowledges the support of the Town of Tralee Vocational Education Committee. We acknowledge useful discussions with Gordon Davies, Eoin O'Reilly and Kevin O'Donnell.

\section{References}

Chandrasekhar H R, Fisher P, Ramdas A K and Rodriguez S 1973 Phys. Rev. B 8 3836-51

Cherlow J M, Aggarwal R L and Lax B 1973 Phys. Rev. B 7 4547-60

Cohen E and Sturge M D 1977 Phys. Rev. B 15 1039-51

Dean P J and Herbert D C 1979 Excitons; Springer Topics in Current Physics vol 14, ed. K Cho (Berlin: Springer) pp 55-182

Henry M O, Lightowlers E C, Killoran N, Dunstan D J and Cavenett B C 1981 J. Phys. C: Solid State Phys. 14 L $255-61$

Hopfield J J, Thomas D G and Lynch R T 1966 Phys. Rev. Lett. $17312-5$

Kirczenow G 1977 Solid State Commun. 21 713-7

Kohn W and Luttinger J M 1955 Phys. Rev. 98 915-25

Labrie D, Timusk T and Thewalt M L W 1984 Phys. Rev. Lett. 52 81-4

Laude L D, Pollak F H and Cardona M 1971 Phys. Rev. B 32623-36

Lipari N O and Baldereschi A 1978 Solid State Commun. 25 665-8

Maeda K 1965 J. Phys. Chem. Solids 26 595-7

Morgan J van W and Morgan T N 1970 Phys. Rev. B $1739-49$

Onton A and Morgan T N 1970 Phys. Rev. B $12592-604$

Rodriguez S, Fisher P and Barra F 1972 Phys. Rev. B $52219-33$

Thewalt M L W, Watkins S P, Ziemelis U O, Lightowlers E C and Henry M O 1982 Solid State Commun. 44 573-8

Wagner J and Sauer R 1982 Physica 117/118B 113-5

Watkins S P, Thewalt M L W and Steiner T 1984 Phys. Rev. B 29 5727-38

Wright G B and Mooradian A 1967 Phys. Rev. Lett. 18 608-10 